\title{
Optimising Outcomes During the Catheter Ablation of Ventricular Tachycardia
}

\author{
An Expert Interview with Jose L Merino \\ La Paz University Hospital, Madrid, Spain
}

DOI: https://doi.org/10.17925/EJAE.2017.03.01.11

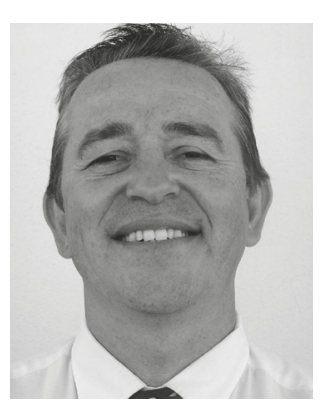

\begin{abstract}
Jose L Merino
Jose L Merino is Director of the Arrhythmia and Robotic Electrophysiology Unit at La Paz University Hospital, Madrid, Spain. His main research interest is treatment of atrial and ventricular arrhythmias, and he has made more than 300 contributions as congress abstracts and has had more than 100 articles published in indexed journals, such as the New England Journal of Medicine and Circulation. His main contributions in this field have been for bundle branch reentry ventricular tachycardia and superior vena cava flutter. He is also involved in the development of new antiarrhythmic and anticoagulant drugs, electroanatomical mapping (EnSiteTM) and remote navigation systems (the Magnetecs Catheter Guidance Control and Imaging system, Amigo-Catheter Robotics) for electrophysiology. He is past President of the Working Group on Arrhythmia (Spanish Society of Cardiology), past Chairman of the Accreditation Committee of the European Heart Rhythm Association (EHRA) of the European Society of Cardiology (ESC), and present Chairman of the Training Fellows Committee of the EHRA. He has been also member of the Scientific Committee of several Europace congresses (in 2007, 2009 and 2011) and has participated actively in live and pre-recorded case transmissions. Furthermore, he is a Fellow of the ESC, an honorary member of the Argentinian Society of Cardiology and a guest of the Venezuela, Peru, Uruguay and Costa Rica societies of cardiology. Professor Merino received his PhD degree (doctoral thesis) and became a cardiologist in 1993. He underwent further training in cardiac arrhythmias
\end{abstract} and electrophysiology (1993-1995) at Gregorio Marañon (Spain), the Boston Children's (US) and London St George's (UK) hospitals.

\section{Keywords}

Catheter ablation, pericardium, mapping, ventricular tachycardia

Disclosure: Jose L Merino has nothing to declare in relation to this article. No funding was received in the publication of this article. This is an expert interview piece and as such has not undergone the journal's standard peer review process.

Acknowledgements: Editorial assistance was provided by Katrina Mountfort, Freelance Medical Writer, supported by Touch Medical Media.

Authorship: The named author meets the International Committee of Medical Journal Editors (ICMJE) criteria for authorship of this manuscript, takes responsibility for the integrity of the work as a whole, and has given final approval to the version to be published.

Open Access: This article is published under the Creative Commons Attribution Noncommercial License, which permits any non-commercial use, distribution, adaptation and reproduction provided the original author(s) and source are given appropriate credit. Received: 7 March 2017

Published Online: 1 June 2017

Citation: European Journal of Arrhythmia

\& Electrophysiology, 2017;3(1):11-2

Corresponding Author: Jose L Merino, La Paz University Hospital, Paseo de la Castellana, 261, 28046 Madrid, Spain. E: jlmerino@arritmias.net
V entricular tachycardia (VT) typically arises from structural heart disease and it is often associated with a high risk of sudden cardiac death. Catheter ablation therapy is a valuable treatment modality for recurrent VT and can reduce the incidence of implantable cardioverter-defibrillator (ICD) shocks, which are painful, decrease quality of life and can cause post-traumatic stress disorder. However, catheter ablation is a challenging procedure that requires advanced technical and manual skills. Procedural complications require prompt intervention. In addition, strategies, methods, and technology are evolving rapidly. In this expert interview, Jose L Merino of Madrid, Spain, discusses the key considerations to optimise outcomes.

\section{Q: What are the key technical considerations when performing epicardial puncture to avoid complications in ventricular tachycardia ablation?}

Complications of epicardial VT ablation could be related to pericardial access and to mapping and ablation within this anatomical structure. Among the former, unintended puncture of the right ventricle (RV) is probably the most frequent (up to 17\%) and commonly does not result in significant consequences. However, the risk of laceration of the ventricular wall or a branch of a coronary artery is always present and may result in severe bleeding and haemodynamic collapse. Therefore, it is important to perform a careful puncture of the pericardial space with the needle being not totally perpendicular to the ventricular wall. Ventricular puncture can be suspected by detection of ventricular ectopic on the electrocardiogram (ECG) or air bubbles or the guide wire artefact in the RV by intracardiac echocardiography (ICE). Another potential severe complication of pericardial access is unintended liver puncture and bleeding. Patients with congestive heart failure or anatomical anomalies are at particular risk and the use of imaging techniques prior to the procedure could be useful.

\section{Q: How can embolism, phrenic palsy and erosion of the coronary arteries be avoided in ventricular tachycardia ablation?}

Cerebral embolism is a severe complication which recently has been found to occur subclinically in many patients after endocardial VT ablation. Presently, it is recommended to use a similar level of anticoagulation to that used for atrial fibrillation ablation (heparine during the procedure to ensure an activated clotting time [ACT]>250-300 ms), especially with the use of long sheaths in the systemic circulation or when multiple radiofrequency (RF) lesions are created. The left phrenic nerve (PN) courses thought the lateral wall of the left ventricle and can be potentially damaged by RF delivery. PN capture by pacing from the ablation catheter can predict this complication. Interposition of a balloon, or infused fluid/air between the catheter and the PN may prevent it. The combined use of a steerable sheath and a contact- 
sensing catheter is recommended to ensure proper RF delivery to the tissue while keeping the PN away. The coronary arteries may also be damaged if RF is delivered less than $5 \mathrm{~mm}$ away and this needs to be checked by coronary angiography.

\section{Q: When should one use retrograde, antegrade, epicardial or robotic approaches in ventricular tachycardia ablation?}

The retrograde and antegrade left ventricle endocardial approaches are mostly used to facilitate outflow track and lateral mapping, respectively. However, there are other considerations such the presence of peripheral artery or aortic valve diseases, which may limit the retrograde approach, or mitral disease or severe left atrium dilatation, which may limit the antegrade approach. The epicardial approach is used whenever the isthmus of slow conduction is suspected to be located in this region, as it is in chagasic or right arrhythmogenic cardiomyopathies. Mapping of this space is becoming a regular procedure in most labs. Finally, robotic navigation allows the operator to easily map the VT substrate without being tied to the patient table. In addition, it is of particular interest for epicardial mapping by using a magnetic system (the Niobe ${ }^{\circledR}$ system [Stereotaxis, St Louis, Missouri, US] or the Magnetecs Catheter Guidance Control and Imaging system), which allows moving the catheter freely within the pericardic sac, which is not the case for conventional mechanically driven catheters, which often need the support of a steerable sheath to reach some areas.

\section{Q: If complications occur during ventricular tachycardia ablations, what are the optimum strategies for their management?}

Early detection of complications is critical. The patient needs to be monitored for blood pressure and oxygen saturation and his or her conscious status should be checked regularly when no deep sedation/anaesthesia is used. The use of ICE provides some benefits in order to improve the success rate (relationship of the ablation catheter with the chamber and papillary muscles, scar detection, etc.) together with live monitoring of complications (thrombus formation, tissue overheating, first signs of pericardial effusion, etc.). Once the complication has been detected, it is recommended to have a well-established, predefined plan with clear steps, which need to be followed by all components of the electrophysiology lab team. The anticoagulation status of the patient needs to be checked and corrective treatments (protamine, prothrombin complex, VIla factor, etc.) should be at hand. Both a cardiology interventional and a cardiac surgery team need to be readily available for early intervention. $\square$ 